\title{
Acceso y permanencia de estudiantes con discapacidad en las universidades chilenas
}

\section{Access and continuity of students with disability in Chilean universities}

\section{Guillermo Andrés Rodríguez MolinA* \\ BÁRBARA VALENZUELA ZAMBRANo**}

El objetivo del artículo es analizar el acceso de estudiantes con discapacidad a las universidades chilenas que se adhieren al proceso regular de admisión, e identificar las barreras para las personas en situación de discapacidad tanto en el sistema de acceso como en los programas de apoyo. La indagación documental presenta un análisis de contenido sobre datos de acceso (en términos de admisión) y visualización de políticas para apoyar la permanencia de las personas en el proceso formativo (acciones o programas) desde lo ideal o prescriptivo. Los hallazgos fueron contrastados con ocho entrevistas semiestructuradas a universitarios que cursan sus carreras en universidades chilenas. Los resultados confirman que la principal barrera para ellos/ as es que no hay criterios comunes entre universidades y los trámites para postular difieren entre instituciones. Como principal conclusión se propone al Estado chileno contar con un único y centralizado sistema de ingreso especial para personas con discapacidad, con cuotas de admisión por universidad asociadas a indicadores objetivos institucionales (infraestructura, materiales, etcétera) para no caer en discriminación arbitraria.

The aim of this article is to analyze the access of students with disability toward Chilean universities which belong to the regular process of admission, identifying the existent barriers to people with a disability situation both in the access system and support programs. It is an inquiring documentary article which presents an analysis of content about access datum (in respect of admission) and politics visualization to support the continuity of people in the formative process (actions or programs) from either ideally or prescriptively. Results which were extrapolated with eight semi-structured interviews to undergraduates who are taking their courses in universities. The results verified that the main barrier for them is the absence of shared criteria among universities and the procedure to apply are different in each institution. It is strongly recommended that the Chilean state takes into account a unique and centralized Special Entry System to people with disability, with admission fees per university associated to objective institutional indicators (Infrastructure, materials, etc.), so as to not fall into arbitrary discrimination.

\section{Palabras clave:}

acceso y

permanencia de universitarios/as, universitarios/as con discapacidad, barreras para la inclusión de personas con discapacidad

Keywords:

access and continuity of undergraduates, undergraduates with disability, barriers against inclusion of people with disability

Recibido: 21 de noviembre de 2018.| Aceptado para su publicación: 25 de junio de 2019 Recuperado de: https://sinectica.iteso.mx/index.php/SINECTICA/article/view/927 doi: 10.31391/S2007-7033(2019)0053-002

Sección: Investigaciones temáticas

* Magíster en Psicología. Decanato Facultad Educación y colaborador académico de la Universidad de Concepción de Chile. Líneas de investigación: educación e inclusión. Correo electrónico: guiandres.rm@gmail.com/ http://orcid.org/0000-0002-3499-1004

** Doctora en Ciencias de la Educación. Profesora asistente en el Departamento de Ciencias de la Educación de la Universidad de Concepción de Chile. Líneas de investigación: educación e inclusión. Correo electrónico: barbaravalenz@gmail.com 


\section{ANTECEDENTES}

$\mathrm{E}$ n los últimos diez años, el acceso de personas con discapacidad a la universidad ha aumentado considerablemente y se espera que este crecimiento siga en ascenso (Abu-Hamour, 2013; Konur, 2006). No obstante, al comparar la educación superior con los anteriores ciclos formativos, se observa que este sigue siendo el ciclo más excluyentes en el que todavía existen muchas barreras para la inclusión (IESALC, 2005); es más, para algunos autores (Lissi et al., 2009) esta situación se debe a que la inclusión en educación superior no ha tenido el impulso que se ha registrado en los otros niveles escolares.

En España, por ejemplo, se observa que las personas con discapacidad acceden en menor proporción $(10.6 \%)$ que las personas que no tienen ninguna discapacidad (28.9\%) (OED, 2016). Australia registra uno de los mejores porcentajes en este ciclo formativo para personas con discapacidad, el cual llega al 21\% (NCSEHE, 2016). En Estados Unidos, el porcentaje entre 2005 y 2010 de personas con discapacidad que asistieron a la universidad alcanzó el 19\% y se estima que las cifras actuales representen el 20\% (Grigal, Hart \& Migliore, 2011).

En Latinoamérica, las cifras son menos alentadoras: en México, la población con discapacidad que obtiene un nivel educativo universitario es de solo el 6\%, porcentaje muy por debajo del $16 \%$ de personas adultas sin discapacidad que cursaron una carrera universitaria (Sedesol, 2016). En Colombia, el porcentaje de personas (de las cinco ciudades con mayor población) que se encuentra cursando estudios de enseñanza superior (técnicos o universitarios) apenas llega al 5.5\% (Minsalud, 2018).

En Chile, si bien los procesos de inclusión educativa en la universidad han aumentado en los últimos años (Lissi et al., 2009), todavía las cifras siguen siendo bajas en relación con la población en edad y posibilidad de estudiar una titulación universitaria. De acuerdo con el Segundo Estudio Nacional de la Discapacidad, existe una diferencia de más del $10 \%$ entre el $20 \%$ de población adulta que ha terminado una titulación, comparado con solo el $9.1 \%$ de personas en situación de discapacidad que logra terminar el ciclo de educación superior (Senadis, 2016).

\section{FUNDAMENTACIÓN}

Acceso de estudiantes con discapacidad a las universidades chilenas

La revisión que aquí presentamos analiza solo el sistema de acceso de personas con discapacidad de aquellas universidades que se adscriben al proceso de admisión regular, que, al término de 2017, son un total de 39 universidades (DEMRE, 2017).

En Chile, los mecanismos de acceso de estudiantes con discapacidad a la universidad no se rigen por un proceso estándar (ni siquiera en aquellas instituciones adscritas al proceso de admisión regular); quedan las vacantes asignadas y las formas de ingreso a criterio de cada institución. Por ello, en la práctica coexisten tres posibles vías de ingreso a la universidad, utilizadas por parte de estudiantes en situación de discapacidad, las cuales se detallan a continuación:

-Por medio de la prueba de selección universitaria (PSU) adaptada: debe ser solicitada previamente al Departamento de Evaluación, Medición y Registro 
Educacional (DEMRE) para que este genere la adecuación del instrumento, en razón de las necesidades físicas o sensoriales del estudiantado. Sin embargo, la PSU actual no es del todo inclusiva, ya que no presenta adaptaciones para personas ciegas y sordo-ciegas, sino solo para personas con baja visión.

-Estudiantes en situación de discapacidad que rinden la prueba PSU sin adaptación: es decir, no informan de su condición previamente y, por tanto, la realizan como los demás estudiantes y en las mismas condiciones.

-Ingreso por sistema de admisión especial: es la vía más utilizada por parte de este colectivo (DEMRE, 2017). Este sistema funciona a través de la solicitud de cupos a ingreso especial ofrecidos por algunas universidades; se someten, entonces, a mecanismos alternativos de admisión diseñados por cada institución.

\section{Permanencia de estudiantes con discapacidad en las universidades chilenas}

En Chile no hay estimaciones de la tasa de permanencia y menos de abandono del estudiantado con discapacidad en las instituciones (Lissi et al., 2013). Esta desinformación afecta la visualización de las necesidades de este colectivo en su camino hacia la obtención de un título profesional, situación que justificaría contar con actualizaciones de cuántos programas de apoyo coexisten en las universidades chilenas y cuáles son sus objetivos de trabajo en relación con el colectivo. Un informe de la Organización de las Naciones Unidas para la Educación, la Ciencia y la Cultura (Unesco), de 2005, daba cuenta de la falta de programas de apoyo, ya que, hasta entonces, solo cinco instituciones (de 50 universidades que se adscribían al proceso de admisión regular) declararon que contaban con un programa de apoyo para estudiantes en situación de discapacidad; es decir, apenas un 10\% del total de universidades que eran parte del proceso de admisión regular en ese año.

Estos programas, además, diferían tanto en su funcionamiento interno como en el tipo de población con discapacidad que apoyaban, ya que algunos beneficiaban solo a estudiantes con discapacidad visual y otros, a estudiantes con problemas motores (IESALC, 2005).

Debido a las condiciones de acceso y permanencia mencionadas, algunos autores señalan que los universitarios chilenos en situación de discapacidad experimentan variadas condiciones de inequidad (Lissi et al., 2009; Valenzuela-Zambrano, Panão, ChacónLópez y López-Justicia, 2017). Al respecto, Vásquez y Alarcón (2016) aseguran que las universidades chilenas, si bien han logrado avances en la declaración de misiones y valores en torno a lo que cada institución considera como inclusión, en muchas ocasiones estas declaraciones no son coherentes con la política y práctica institucional, al existir vacíos y contradicciones tanto entre instituciones como al interior de cada una.

Cabe entonces preguntarse ¿cuáles son esos obstáculos o barreras que estos/as estudiantes chilenos atraviesan tanto en el acceso como en la permanencia y que no padecen sus pares sin discapacidad? Por ello, este estudio tiene como objetivo analizar el acceso de estudiantes con discapacidad a las universidades chilenas que se adhieren al proceso regular de admisión, e identificar las principales barreras para las personas en situación de discapacidad en el sistema de acceso y en los programas de apoyo desde su propia experiencia. 


\section{MARCO TEÓRICO}

\section{Barreras para la inclusión universitaria}

Se les denomina barreras para la inclusión a aquellas variables (del individuo o del ambiente) que impiden o inhiben la participación plena en la sociedad. Algunos autores señalan dos importantes barreras para la inclusión educativa: las de acceso (infraestructura y espacios) y las de acceso al currículo (todos los aspectos que tengan que ver con los obstáculos que dificultan la dinámica de enseñanza-aprendizaje) (Borland \& James, 1999; Konur, 2006).

Darrow (2009), por su parte, propone tres barreras:

-Barreras organizacionales: se relacionan con la institución educativa en cuanto a la estructura organizacional; también se refieren a la ausencia de políticas que visibilicen la inclusión como sello institucional, lo que dificulta la participación efectiva de este colectivo.

-Barreras culturales: son los estereotipos, prejuicios y actitudes relacionados con el fenómeno como tal y que, por ser no observables a veces, pueden ser muy influyentes (Gitlow, 2001).

-Barreras de conocimiento: dimensión asociada a las prácticas educativas propiamente tales, y cómo estas pueden transformarse en barreras cuando reflejan aspectos de la política y la cultura que pueden llegar a materializarse cuando no son intervenidos en actos concretos de discriminación arbitraria.

En el caso de las barreras relacionadas con el acceso a la universidad por parte de personas con discapacidad, algunos autores han resaltado las falencias del propio proceso de admisión, cuando este no entrega una información completa sobre cómo postular, en qué institución y los cupos designados para cada carrera (Borland \& James, 1999; Salinas, Lissi, Medrano, Zuzulich y Hojas, 2013).

Un informe reciente realizado entre el Centro Interuniversitario de Desarrollo (CINDA) y el Grupo Operativo de Universidades Chilenas tuvo como objetivo revisar y proponer orientaciones en inclusión en educación superior. Este informe señala que, si bien se destacan las buenas prácticas de las instituciones chilenas, estas se encuentran fundamentalmente en el nivel operativo, de ahí que en el tema del acceso propone revisar la manera en que acceden y cómo las instituciones apoyan al estudiantado una vez aceptado, pues se presentan discordancias entre las política nacional impulsada por el Estado chileno en la materia y lo que sucede en cada institución respecto a las prácticas efectivas que surgen de la interpretación del modelo de inclusión que hace cada institución (CINDA, 2019).

Esta barrera en el acceso ya había sido expuesta dos años atrás, en un estudio de revisión documental que comparaba las políticas de acceso y permanencia de estudiantes universitarios portugueses con las chilenas (Valenzuela-Zambrano et al., 2017); la principal falencia en el proceso de admisión de este último era no contar con un sistema centralizado de admisión especial como el que opera en Portugal.

En cuanto a las barreras que dificultan la permanencia, Medrano (2009), en un estudio cualitativo sobre la inclusión en instituciones de educación superior chilenas, encontró como principales barreras la falta de información de los docentes respecto 
a cómo tratar y adecuar sus metodologías de enseñanza para personas con discapacidad, así como también dificultades de los propios estudiantes para enfrentar las exigencias académicas de la universidad.

Esta falta de conocimiento por parte de los docentes universitarios de cómo apoyar la inclusión de estudiantes con discapacidad en sus aulas es ratificada en un estudio nacional en el cual se entrevistaron estudiantes con discapacidad de distintas instituciones y que señalaron como obstáculo para avanzar en sus estudios no solo la falta de conocimiento, sino las actitudes de poca cooperación de algunos profesores (Salinas et al., 2013).

En la misma línea, otro estudio nacional, que realizó una encuesta a 38 estudiantes con discapacidad, resalta que una de las principales barreras es la falta de articulación de las iniciativas de la universidad para favorecer el acceso y la permanencia de personas con discapacidad (los programas) con instancias concretas de capacitación para sus profesores y administrativos en la materia (Villafañe, Corrales y Soto, 2014).

Un estudio reciente que indagó sobre las barreras percibidas por parte de estudiantes tutores de estudiantes con discapacidad señala entre sus resultados que la conceptualización individual que se tenga de discapacidad puede generar prácticas más o menos inclusivas, por lo que es, a veces, una barrera y en otras experiencias, un facilitador de la inclusión (Victoriano, 2017).

Este aspecto ratifica lo señalado por Echeita, Simón, López y Urbina (2013), quienes mencionan que "a pesar de los esfuerzos que se hagan para que la educación inclusiva sea una realidad, los variados significados del concepto que se le den pueden producir obstáculos para la propia realización de esta" (p. 104). De lo expuesto se desprende que la función de los programas de apoyo es fundamental para educar a los distintos actores universitarios (estudiantes, docentes, personal) en un modelo de concepción de la discapacidad actualizado (basado en enfoque de derechos) y que, a su vez, sea compartido por las distintas instituciones de educación superior.

Otra barrera citada por algunas investigaciones e informes en la materia en cuanto a la permanencia es que se advierte una falta de asignación presupuestaria destinada al apoyo de los estudiantes con discapacidad, sin hacer distinciones del resto de la población universitaria en cuanto a los requerimientos y las necesidades para el logro de los objetivos académicos (Lissi et al., 2009; Valenzuela-Zambrano et al., 2017; CINDA, 2019). Esto no deja de ser un factor importante a considerar, ya que la estimación de gastos de un universitario chileno con discapacidad es tres veces mayor que el de un estudiante sin discapacidad (Zuzulich, Achiardi, Hojas y Lissi, 2014). Estos gastos son subvencionados, en gran parte, por las propias instituciones y algunos recursos parciales que otorga el Estado a través de los concursos anuales de ayudas técnicas y apoyo para la creación de unidades de inclusión universitarias, por medio del Servicio Nacional de la Discapacidad.

Todos estos aspectos hacen necesaria una revisión de las políticas de acceso y permanencia declaradas por las universidades chilenas que se adhieren al proceso de admisión regular para identificar las barreras que se siguen manteniendo respecto al acceso y la permanencia de estudiantes con discapacidad para luego contrastar esa información oficial con la experiencia de los propios involucrados/as. 


\section{METOdología}

Nuestro estudio es de corte cualitativo y está conformado por una primera indagación documental de consulta de documentos oficiales del proceso de admisión regular y de los portales institucionales que describen las políticas para apoyar la permanencia de personas con discapacidad (acciones o programas). Estos resultados alimentaron el proceso de construcción del guion de las entrevistas semiestructuradas realizadas, las cuales fueron trabajadas a través del análisis de contenido con la finalidad de efectuar deducciones lógicas y justificables concernientes a la fuente (el emisor y su contexto) (Bardin, 1986, citado en López, 2012).

\section{Participantes}

La muestra intencionada se integró de ocho estudiantes: cinco hombres (dos con discapacidad motora, otro con baja visión y uno diagnosticado con trastorno del espectro autista) y tres mujeres (dos con discapacidad motora y una con discapacidad auditiva parcial), con un promedio de edad de veinticuatro años y pertenecientes a tres universidades de la Región del Bío Bío, Chile. El criterio de inclusión fue poseer una condición de discapacidad y ser alumno/a regular. También es importante señalar que el tamaño de la muestra se definió considerando la saturación teórica de las categorías (Flick, 2004).

\section{Instrumentos}

Para la primera parte del estudio se revisó el documento oficial número 4 del DEMRE, que señala la oferta definitiva de carreras, vacantes y ponderaciones del proceso de admisión 2018, además de los 36 portales institucionales de las universidades (públicas y privadas) que se adscriben al proceso de admisión regular (DEMRE, 2017).

A partir del análisis documental, las categorías emergentes se utilizaron para construir el guion de una entrevista semiestructurada, que buscó indagar los temas de acceso y permanencia en la universidad, así como las principales barreras u obstáculos por ellos/as experimentados asociados a su situación de discapacidad. Las entrevistas consideraron una secuencia temporal desde cuarto año medio (año en que termina la enseñanza media) hasta la actualidad en sus procesos formativos universitarios. Para asegurar la fiabilidad intersubjetiva en el tratamiento de los datos, ambos investigadores realizaron codificaciones paralelas de todas las entrevistas (Flick, 2004).

\section{Procedimiento}

Nuestro estudio se llevó a cabo en dos etapas. La primera consistió en una revisión documental que alimentó el proceso de construcción de la entrevista, y que determinó las temáticas y preguntas directrices. Posteriormente, hicimos una invitación a los estudiantes de la región por correo electrónico para informarles acerca de los objetivos y aspectos éticos del estudio. Luego, efectuamos las entrevistas semiestructuradas, 
previa lectura de consentimiento informado que autorizaba la grabación de audio y explicaba el carácter voluntario y anónimo de la participación en ella.

\section{RESULTADOS}

Como el estudio está compuesto por dos etapas, en primer lugar presentamos los resultados del análisis documental, en cuanto a las barreras que afectan el acceso a la universidad y, en seguida, los resultados encontrados en las barreras para la permanencia en la universidad. Hacemos la misma división en las categorías referentes a las barreras que tienen que ver con el acceso y luego con la permanencia.

\section{Análisis documental}

Barreras para el acceso de estudiantes con discapacidad en las universidades chilenas

En la tabla 1 (ver al final del documento) se presentan las universidades que, a septiembre de 2017, declararon contar con procesos de admisión especial para personas con discapacidad (DEMRE, 2017); señalaron si esta admisión pone condiciones según el tipo de discapacidad y si las bases para postular están disponibles en los portales.

Al revisar los datos recogidos en la tabla 1, es posible observar en primer lugar que 23 de las 39 instituciones (58.9\%) que se adscriben al proceso de admisión regular universitario cuentan con sistemas de admisión especial para estudiantes con discapacidad, es decir, un 40\% todavía no tienen sistema de admisión para este colectivo.

También los 23 sistemas de admisión especial existentes son diferentes entre sí; por ejemplo, hay seis programas de admisión especial que son exclusivos para personas con discapacidad visual (no videntes o ciegas, algunos de ellos permiten el acceso a personas con baja visión); dos que permiten participar a estudiantes con discapacidad sensorial o motora; y tan solo ocho programas no distinguen por tipo de discapacidad presentada.

Por ello, contar con tanta amplitud de criterios respecto al tipo de discapacidad con la que se puede, o no, acceder por vía de admisión especial, así como la restricción de titulaciones, ponen a los estudiantes con discapacidad en una situación de desventaja, pues limitan sus posibilidades de escoger según sus intereses y preferencias vocacionales, lo que constituye un obstáculo a la inclusión universitaria; esto es confirmado con datos presentados en otros estudios internacionales y nacionales (Borland \& James, 1999; Salinas et al., 2013; Valenzuela-Zambrano et al., 2017, CINDA, 2019).

Por lo expuesto, es posible enumerar la existencia de tres barreras en el acceso de personas con discapacidad al sistema universitario chileno:

- No se cuenta con un único sistema de admisión especial para personas con discapacidad que rija en la totalidad de las instituciones de educación superior.

-Los sistemas de admisión especial existentes presentan una variabilidad de criterios para postular a ellos y algunos también restringen los cupos de acceso a determinadas titulaciones, lo que dificulta el acceso en condiciones de equidad para este colectivo. 
-La información para el proceso de postulación al sistema de admisión especial en algunas instituciones no se encuentra disponible en los portales institucionales.

Barreras para la permanencia de estudiantes con discapacidad en las universidades chilenas

La tabla 2 (ver al final del documento) fue elaborada a partir de la revisión de los portales institucionales de las 39 universidades que se adscribieron al proceso de admisión regular 2018, y de la consulta del boletín oficial número 4 del DEMRE, que reglamenta el proceso de admisión regular 2018 (DEMRE, 2017). La tabla ofrece información sobre la presencia de programas institucionales para apoyar la permanencia de personas con discapacidad, junto con sus respectivas declaraciones de objetivos de trabajo y público a quien está dirigido.

En una primera lectura es posible destacar la amplitud de propuestas de trabajo institucionales con que se aborda un mismo fenómeno, con base en las cuales también se infiere cómo cada institución entiende el trato hacia las situaciones de discapacidad; ejemplo de ello lo constituye la Universidad Tecnológica Metropolitana, que no cuenta con un programa de apoyo directo como tal, sino con un centro de cartografía táctil que provee de recursos tecnológicos (representaciones cartográficas tridimensionales) a personas con discapacidad visual, principalmente; o el caso de la Universidad Metropolitana de Ciencias de la Educación, cuyo programa es una central de recursos pedagógicos para la inclusión, que apoya a los docentes que trabajan con estudiantes en situación de discapacidad.

Esta variabilidad de criterios puede ser un reflejo de las necesidades propias de cada institución, pero también puede generar confusión, es decir, actuar como facilitador o barrera de la inclusión, como algunos autores han planteado (Echeita et al., 2013; Victoriano, 2017).

También es posible apreciar que la mayoría de las instituciones trabaja solo con los estudiantes con discapacidad, pero pocos declaran hacer trabajo con el resto de la comunidad universitaria, sobre todo si investigaciones ya han ratificado que la falta de coordinación entre el programa de apoyo y los docentes puede ser una barrera importante (Medrano, 2009; Salinas et al., 2013; Villafañe et al., 2014).

Por ello, como principales barreras en la permanencia el análisis documental entrega dos:

-Falta de criterios unificados de trabajo entre los programas de apoyo; es necesario ampliar su cobertura a todo tipo de situaciones de discapacidad en algunas instituciones.

-Pocos programas incluyen al resto de la comunidad educativa y no aportan a una concepción del fenómeno de la discapacidad compartido trasversalmente. 


\section{Análisis de las entrevistas}

Barreras para el acceso de estudiantes con discapacidad en las universidades chilenas

En los relatos de los estudiantes entrevistados advertimos sobre todo barreras organizacionales, pero también culturales. Las primeras guardan relación con la falta de criterios de acceso unificados por parte de las instituciones universitarias, lo que restringe las posibilidades de elección respecto a sus pares sin discapacidad, resultados que confirman lo evidenciado en la revisión de documentos oficiales:

El año pasado entré a otra carrera en Los Ángeles, pero siempre me gustó Arquitectura, no me atreví a postular ese año, pero al final me salí de la otra carrera, porque no me gustó [...] Sí se me ha hecho difícil estar lejos, pero allá no está Arquitectura (entrevistado 4).

Yo quería estudiar Educación Diferencial, pero ese año no quedé en el cupo especial, quedo otra niña y para no perder el año me metí a Básica (entrevistada 3).

En cuanto a las barreras culturales, existe una percepción de exclusión por causa de su situación de discapacidad en cinco de los ocho entrevistados/as. De acuerdo con la literatura revisada, estas barreras, al no ser tangibles, no pueden vislumbrarse en la revisiones documentadas, pero sí es posible visibilizarlas a través de los relatos de los propios afectados. En estos casos, las personas que hicieron sentir esta percepción de exclusión o rechazo se refieren a personal universitario y a sus compañero/as. Cabe destacar que no se dieron casos asociados a actitudes negativas por parte de docentes:

Te has sentido discriminado arbitrariamente en la Universidad por tu discapacidad (entrevistador).

Sí, cuando estaba postulando a la ayudantía de Química general. Lo intenté dos veces y la última vez que lo intenté fui a hablar con la secretaria, que es la encargada de elegir a las personas, y me dio a entender que prácticamente yo no podía quedar porque estaba en silla de ruedas, como que no tenía las mismas capacidades que cualquier otra persona (entrevistado 1).

Sí me he sentido discriminada cuando hacemos grupos de trabajo y por ejemplo tenemos que disertar, como todos saben que me cuesta hablar, me cuesta que un grupo quiera trabajar conmigo (entrevistada 2).

Una vez me pasó, que le pedí a la Jefa Administrativa de mi Facultad un espacio para guardar la silla eléctrica y así no depender tanto de mi mamá para trasladarme y ella me dijo que no había espacio [...] ¿Y si hago una carta al Decano? Me dijo, te va a contestar lo mismo que yo, pero me lo dijo en tono pesado, ahí me sentí mal, porque yo sé que es cosa de mala voluntad, no que no se pueda (entrevistada 3).

No en la parte académica, pero quizás un poco incluirme en el entorno universitario, me ha costado socializar en otros ámbitos no académicos con mis compañeros, no me invitan a carretear, a lo mejor pensarán que diré que no (entrevistado 6).

Barreras para la permanencia de estudiantes con discapacidad en las universidades chilenas

Se evidencian barreras arquitectónicas o infraestructura accesible, aunque solo un entrevistado lo declaró:

Todavía no pintan las malditas bajadas de los escalones, voy a escribir una carta a la U porque hay escalones que como veo en blanco y negro, puedo caerme (entrevistado 5). 
Cabe destacar la mayor presencia de barreras de acceso al currículo, que refleja la falta de preparación de algunos docentes para atender la diversidad de personas con discapacidad:

Me he visto afectada más en la parte de las pruebas de que por ejemplo en contabilidad ponen un espacio muy chico, yo no alcanzo a escribir o me falta tiempo, más que nada en la parte de evaluación falta mucho (entrevistada 2).

Creo que le falta cambiar un poco la modalidad de calificar las pruebas y ese tipo de cosas, ver una manera más apropiada para cada discapacidad (entrevistada 8).

Me gustaría que los profesores usen micrófonos, sería muy feliz de poder escuchar toda la clase, además pienso que a todos les beneficiaria (entrevistada 7).

Otra barrera relacionada con el acceso al currículo que se presenta es la rigidez de las mallas curriculares para atender las necesidades educativas especiales en educación superior:

Por ejemplo, un estudiante, por lo general, toma cinco o más ramos por semestre; yo actualmente estoy tomando tres ramos no más, y me arriesgo porque si no me alcanzan los créditos, empiezo a peligrar la posibilidad de que me echen de la carrera, con solamente tres ramos (entrevistado 7).

Algunos estudiantes también denotan falta de conocimientos de los programas de apoyo que hay al interior de sus propias instituciones, u otros no saben qué apoyos entregan. No obstante, hay una valoración de estos espacios como necesarios:

No, no conozco el Programa de la universidad para discapacitados, me gustaría conocerlo y que me ayudara [...] pediría apoyo de especialistas para poder superar todos los problemas que conlleva tener dificultades auditivas como la depresión, ansiedad [...] (entrevistada 7).

No sé si eso [pintar señaléticas en escaleras], lo ve el Programa XXX o tengo que enviar la carta a Servicios? (entrevistado 5).

\section{CONCLUSIONES Y DISCUSIÓN}

Este estudio aporta una aproximación a los ajustes necesarios en las políticas y en las prácticas de inclusión de personas con discapacidad en el ámbito universitario. En relación con el acceso, la ausencia de un consenso entre universidades para regular el proceso de admisión especial de este colectivo es el principal obstáculo, visualizado en la normativa, y también padecido por los estudiantes; diversos estudios ya lo han corroborado (Borland \& James, 1999; Salinas et al., 2013; Valenzuela-Zambrano et al., 2017, CINDA, 2019). Por ello, es necesario consensuar políticas estatales que permitan contar con un único sistema de ingreso especial para estudiantes con discapacidad que unifique los criterios para postular a él y que se eliminen las restricciones de acceso a determinadas carreras o titulaciones, que solo promueven condiciones de inequidad (Lissi et al., 2009; Lissi et al., 2013; Valenzuela-Zambrano et al., 2017; Zuzulich et al., 2014). En virtud de la disparidad de realidades que da cuenta el análisis documental entre instituciones universitarias en la materia, proponemos que este sistema unificado establezca cuotas de ingreso previas por cada institución que consideren la realidad del 
modelo de inclusión institucional (funcionamiento de programas de apoyo, infraestructura accesible, capacitación del profesorado, etcétera).

En cuanto a la permanencia de los estudiantes con discapacidad en las universidades, los resultados del análisis documental y la ratificación de ello en las entrevistas ponen de manifiesto la necesidad de que los programas de apoyo institucionales adopten una decisión sobre criterios de trabajo.

Al igual que en el caso del acceso, los programas de apoyo requieren consensuar sus prácticas y compartir sus experiencias de inclusión, con la finalidad de que los nuevos programas aprendan de las experiencias ya realizadas. Una acción concreta para lograr este propósito sería la creación de una página web (accesible) por parte del Ministerio de Educación Chileno, cuyo principal objetivo sea compartir experiencias interservicios en torno a la inclusión, a través de la difusión de normativas, apoyos y servicios que cada institución de educación superior realice con sus estudiantes.

También es importante trabajar en la coordinación entre el programa de apoyo y lo docentes, ya que la falta de trabajo coordinado fue ratificado en la entrevistas como una barrera para la inclusión y en la línea de las propuestas de estudios nacionales e internacionales (Medrano, 2009; Salinas et al., 2013; Villafañe et al., 2014).

Como futuras líneas de investigación, sugerimos incluir entrevistas de docentes, personal universitario y estudiantes sin discapacidad, ya que los procesos de inclusión afectan a toda la comunidad educativa.

Finalmente, es importante destacar que ningún proceso de inclusión está completo si no se trabaja con el empoderamiento de las personas que se requiere apoyar, en este caso los estudiantes en situación de discapacidad. Al respecto, Ocampo (2012) señala que solo con la participación activa e implicación de los universitarios con discapacidad en los valores del colectivo estudiantil se lograría erradicar las barreras simbólicas presentes en las instituciones de educación superior. Esta propuesta también es rescatada por Charlton (1998), que recoge en la frase "Nada acerca de nosotros sin nosotros" (p. 17) como la forma de impulsar a las personas con discapacidad a hacerse responsables de participar en el sistema político, económico y cultural, relacionado con la toma de decisiones en temas que les atañen.

Es imprescindible, por tanto, que en las reformas de los procesos de acceso y permanencia se consulte a los propios estudiantes con discapacidad (Salinas et al., 2013; Villafañe et al., 2014; CINDA, 2019), pues la responsabilidad de generar los cambios necesarios en la materia es conjunta (estudiantes, universidades y Estado). 


\section{REFERENCIAS BIBLIOGRÁFICAS}

Abu-Hamour, B. (2013). Faculty attitudes toward students with disabilities in a public university in Jordan. International Education Studies, vol. 12, núm. 6, pp. 74-81. Recuperado de https://files.eric.ed.gov/fulltext/EJ1068696.pdf

Borland, J. \& James, S. (1999). The learning experience of students with disabilities in higher education. A case study of a UK university. Disability \& Society, vol. 14, núm. 1, pp. 85-101.

Charlton, J. (1998). Nothing about us without us. Berkeley, CA, EUA: University of California Press.

CINDA (2019). Educación superior inclusiva. Santiago, Chile: Gestión Universitaria.

Darrow, A. (2009). Barriers to effective inclusion and strategies to overcome them. General Music Today, vol. 22, núm. 3, pp. 29-31. Recuperado de http://citeseerx. ist.psu.edu/viewdoc/download?doi=10.1.1.898.7575\&rep=rep1\&type=pdf

DEMRE (2017). Proceso de admisión 2018: universidades del Consejo de Rectores y adscritas al proceso de admisión ( $\left.N^{\circ} 4\right)$. Recuperado de http://psu. demre.cl/publicaciones/2017/2017-16-09-22-cruch-oferta-carreras-vacantes-ponderaciones

Echeita, G., Simón, C., López, M. y Urbina, C. (2013). Educación inclusiva, sistemas de referencia, coordinadas y vórtices de un proceso dilemático. En M. A. Verdugo y R. Schalock (coords.). Discapacidad e inclusión. Manual para la docencia. Salamanca, España: Amarú Ediciones.

Flick, U. (2004). Introducción a la investigación cualitativa. Madrid, España: Morata.

Gitlow, L. (2001). Occupational therapy faculty attitudes toward the inclusion of students with disabilities in their educational programs. The Occupational Journal of Research, vol. 21, núm. 2, pp. 115-131. doi. org/10.1177/153944920102100206

Grigal, M., Hart, D. \& Migliore, A. (2011). Comparing the transition planning, postsecondary education, and employment outcomes of students with intellectual and other disabilities. Career Development and Transition for Exceptional Individuals Journal. Recuperado de http://bcn.cl/20rpq

IESALC (2005). Informe final: integración de las personas con discapacidad en la educación superior en Chile. Recuperado de http://sid.usal.es/idocs/F8/ FD012677/integracion_educacion_superior_chile.pdf

Konur, 0. (2006). Teaching disabled students in higher education. Teaching in Higher Education, vol. 3, núm. 11, pp. 351-363. Recuperado de http://citeseerx. ist.psu.edu/viewdoc/download?doi=10.1.1.531.9916\&rep=rep1\&type=pdf

Lissi, R., Zuzulich, S., Hojas, A., Achiardi, A., Salinas, M. y Vásquez, A. (2013). En el camino hacia la educación superior inclusiva en Chile. Santiago, Chile: Salesianos.

Lissi, R., Zuzulich, S., Salinas, M., Achiardi, A., Hojas, A. y Pedrals, N. (2009). Discapacidad en contextos universitarios. Calidad de la Educación, núm. 30, 306324. doi: http://dx.doi.org/10.31619/caledu.n30.183

López, F. (2012). El análisis de contenido como método de investigación. Revista de Educación, vol. XXI, núm. 4, pp. 167-179. Recuperado de http://rabida. uhu.es/dspace/bitstream/handle/10272/1912/b15150434.pdf?seq

Medrano, D. (2009). Estudiantes universitarios con discapacidad: análisis de los relatos de sus vivencias en el ámbito educativo. Tesis de magíster. Pontificia Universidad Católica de Chile, Santiago de Chile. 
Minsalud (2018). Observatorio Nacional de la Discapacidad. Recuperado de https://www.minsalud.gov.co/sites/rid/Lists/BibliotecaDigital/RIDE/DE/ PS/boletin-9-discapacidad.pdf

NCSEHE (2016). Student equity performance in Australian higher education. Recuperado de http://bcn.cl/20uit

Observatorio Estatal de Discapacidad (OED) (2016). Informe Olivenza sobre la situación y evolución de la discapacidad en España. Recuperado de http://bcn.cl/20qwj

Ocampo, A. (2012). Inclusión de estudiantes en situación de discapacidad a la educación superior. Desafíos y oportunidades. Revista Latinoamericana de Educación Inclusiva, vol. 6, núm. 2, pp. 227-239. doi:10.13140/2.1.2883.0088

Salinas, M., Lissi, M., Medrano, D., Zuzulich, M. y Hojas, A. (2013). La inclusión en la educación superior: desde la voz de estudiantes chilenos con discapacidad. Revista ibero-americana de educação, vol. 63, pp. 77-98.

Secretaría de Desarrollo Social (Sedesol) (2016). Diagnóstico sobre la situación de las personas con discapacidad en México. Recuperado de https://www.gob. $\mathrm{mx} / \mathrm{cms} /$ uploads/attachment/file/126572/Diagn_stico_sobre_la_Situaci_n_ de_las_Personas_Con_Discapacidad._Mayo_2016.pdf

Senadis (2016). Concurso Nacional de Proyectos para Inclusión Social de Personas en Situación de Discapacidad. Recuperado de http://www.senadis.gob. cl/pag/299/1553/concurso_nacional_proyectos_educacion_2016_cerrado

Valenzuela-Zambrano, B., Panão, A., Chacón-López, H. y López-Justicia, M.D. (2017). Alumnado con discapacidad en educación superior en Chile y Portugal: una revisión de las políticas y prácticas que fomentan la cultura inclusiva. Dedica. Revista de Educação e Humanidades, vol. 11, pp. 47-60. Recuperado de file:///C:/Users/baval/Downloads/Dialnet-AlumnadoConDiscapacidadEnEducacionSuperiorEnChileY-5906267.pdf

Vásquez, B. y Alarcón, E. (2016). La inclusión en las universidades chilenas: del discurso a las interacciones prácticas, políticas y culturales. Revista de Investigación Educacional Latinoamericana, vol. 53, núm. 2, pp. 1-19. Recuperado de http://pensamientoeducativo.uc.cl/index.php/pel/article/download/876/1781

Victoriano, E. (2017). Facilitadores y barreras del proceso de inclusión en educación superior: la percepción de los tutores del programa Piane-UC. Estudios Pedagógicos, vol. XLIII, núm. 1, pp. 349-369. Recuperado de https://scielo. conicyt.cl/pdf/estped/v43n1/art20.pdf

Villafañe, G., Corrales, A. y Soto, V. (2014). Estudiantes con discapacidad en una universidad chilena: desafíos de la inclusión. Revista Complutense de Educación, vol. 27, núm. 1, pp. 353-372. Recuperado de file://C:/ Users / baval / D ownloads / 46509-Texto\% 20 del\% 20 art\% C $3 \%$ ADcu lo-94476-1-10-20160114\%20(1).pdf

Zuzulich, M. S., Achiardi, C., Hojas, A. M., y Lissi, M. R. (2014). Inclusión de estudiantes con discapacidad en universidades chilenas: mecanismos de admisión y recursos para su implementación. En Centro de Políticas Públicas UC (eds.). Propuestas para Chile. Concurso políticas públicas (pp. 55-88). Santiago, Chile: Salesianos Impresores. 
Tabla 1. Admisión especial de estudiantes en situación de discapacidad en universidades chilenas

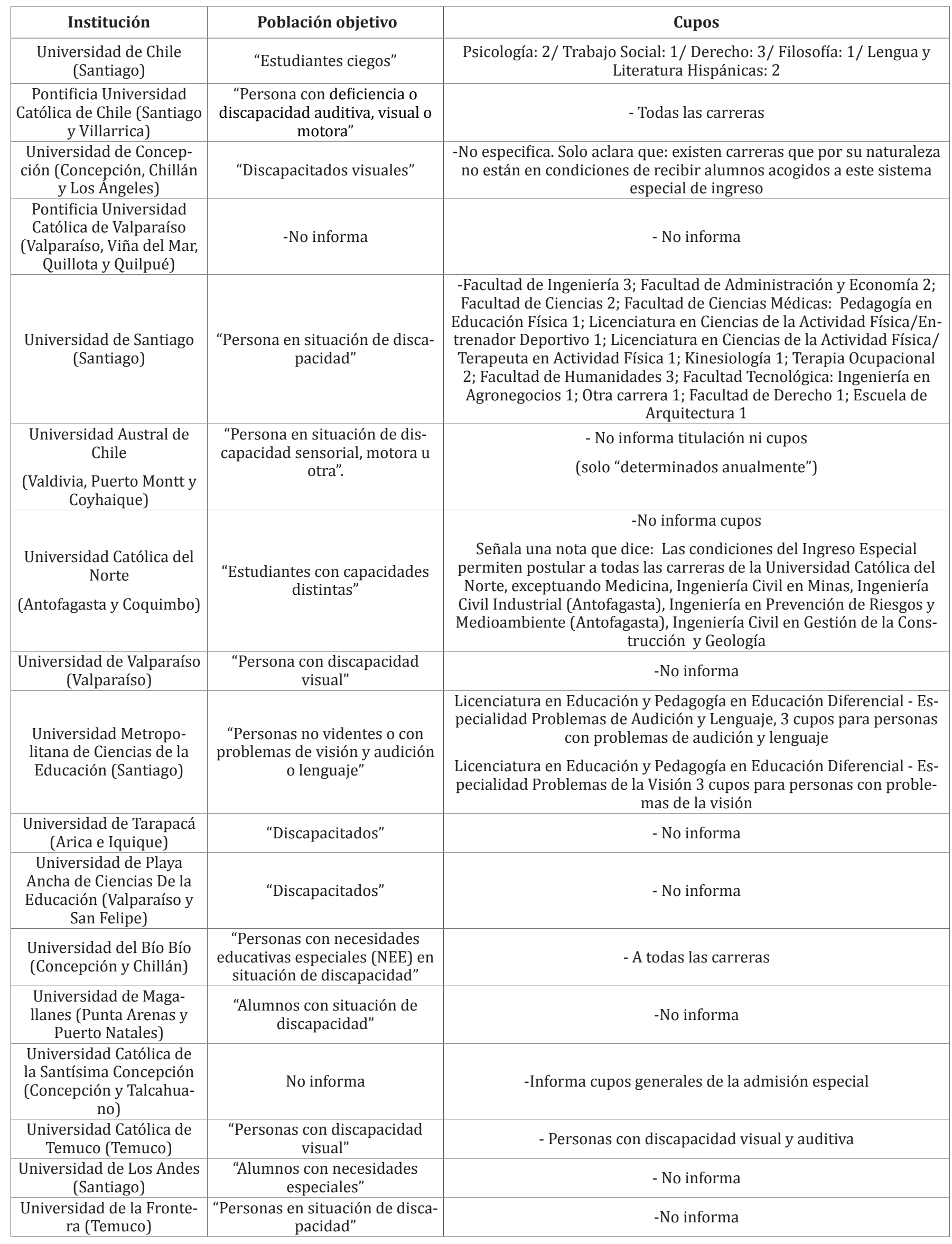




\begin{tabular}{|c|c|c|}
\hline $\begin{array}{c}\text { Universidad Católica del } \\
\text { Maule (Talca y Curicó) }\end{array}$ & $\begin{array}{c}\text { "Personas con discapacidad } \\
\text { visual y física" }\end{array}$ & -Solo carreras Educación Especial y Psicología \\
\hline $\begin{array}{c}\text { Universidad de Aysén } \\
\text { (Coyhaique) }\end{array}$ & $\begin{array}{c}\text { "Jóvenes con discapacidad } \\
\text { motora, visual o auditiva" }\end{array}$ & -No informa \\
\hline $\begin{array}{c}\text { Universidad Diego Porta- } \\
\text { les (Santiago) }\end{array}$ & $\begin{array}{c}\text { "Personas en situación de } \\
\text { discapacidad motora, visual o } \\
\text { auditiva" }\end{array}$ & $\begin{array}{c}\text {-Solo a las carreras de Psicología, Ingeniería Comercial y Derecho (no } \\
\text { informa cupos) }\end{array}$ \\
\hline $\begin{array}{c}\text { Universidad Nacional An- } \\
\text { drés Bello (Santiago, Viña } \\
\text { del Mar y Concepción) }\end{array}$ & $\begin{array}{c}\text { "Personas en situación de disca- } \\
\text { pacidad" }\end{array}$ & -No informa \\
\hline $\begin{array}{c}\text { Universidad Católica Silva } \\
\text { Henríquez (Santiago) }\end{array}$ & $\begin{array}{c}\text { "Personas en situación de disca- } \\
\text { pacidad" }\end{array}$ & -No informa \\
\hline $\begin{array}{c}\text { Universidad San Sebastián } \\
\text { (Santiago, Concepción y } \\
\text { Valdivia) }\end{array}$ & $\begin{array}{c}\text { Estudiantes con situación de } \\
\text { discapacidad }\end{array}$ & -No informa \\
\hline
\end{tabular}

Fuente: elaboración propia desde páginas electrónicas institucionales.

Tabla 2. Programas de apoyo para la permanencia de estudiantes en situación de discapacidad en universidades chilenas adscritas al proceso de admisión regular 2018

\begin{tabular}{|c|c|c|}
\hline Institución & Nombre & Principales objetivos de trabajo y apoyos \\
\hline $\begin{array}{l}\text { Universidad de Chile } \\
\text { (Santiago) }\end{array}$ & $\begin{array}{l}\text { Programa de Apoyo a Estudiantes en } \\
\text { Situación de Discapacidad (PAED) }\end{array}$ & $\begin{array}{l}\text { - Apoyar el ingreso, la permanencia y egreso de estudiantes en } \\
\text { situación de discapacidad (UCHILE, 2017) }\end{array}$ \\
\hline $\begin{array}{l}\text { Pontificia Universidad } \\
\text { Católica de Chile (Santiago } \\
\text { y Villarrica) }\end{array}$ & $\begin{array}{l}\text { Es el Programa para la Inclusión de } \\
\text { Alumnos con Necesidades Especiales } \\
\text { de la UC (PIANE) }\end{array}$ & $\begin{array}{c}\text { - Favorecer la participación de los alumnos con discapacidad } \\
\text { en la Universidad Católica, además de contribuir a generar } \\
\text { conocimientos y estrategias en los docentes para implementar } \\
\text { adecuaciones curriculares no significativas (entre otros } \\
\text { objetivos) (PUC, 2017) }\end{array}$ \\
\hline \multirow[b]{2}{*}{$\begin{array}{l}\text { Universidad de Concepción } \\
\text { (Concepción, Chillán y Los } \\
\text { Ángeles) }\end{array}$} & $\begin{array}{l}\text { El programa interdisciplinario por la } \\
\text { inclusión (INCLUDEC) }\end{array}$ & $\begin{array}{l}\text { - Apoyar la inclusión y permanencia de estudiantes que presen- } \\
\text { ten algún tipo de discapacidad. También, contribuir en el diseño } \\
\text { e implementación de sistemas de acceso especial de personas } \\
\text { con discapacidad (entre otros objetivos) (UDEC, 2017) }\end{array}$ \\
\hline & $\begin{array}{l}\text { Programa de Asistencia Psicopeda- } \\
\text { gógica, Tecnológica, Comunicacional, } \\
\text { Biológica y Psicosocial para Estu- } \\
\text { diantes con Discapacidad Visual de la } \\
\text { Universidad de Concepción (ARTIUC) }\end{array}$ & $\begin{array}{l}\text { - Contribuir con orientaciones didácticas en adaptaciones } \\
\text { curriculares a las características visuales de los estudiantes, } \\
\text { procurar un desarrollo integral en las áreas social, educativa, } \\
\text { psicológica, comunicacional de los estudiantes con discapaci- } \\
\text { dad visual (entre otros objetivos) (UDEC, 2017) }\end{array}$ \\
\hline $\begin{array}{l}\text { Pontificia Universidad } \\
\text { Católica de Valparaíso } \\
\text { (Valparaíso, Viña del Mar, } \\
\text { Quillota y Quilpué) }\end{array}$ & El Programa PUCV Inclusiva & $\begin{array}{l}\text { - Sensibilizar a la comunidad académica en torno a la visibiliza- } \\
\text { ción de barreras para el aprendizaje y el apoyo académico a los } \\
\text { estudiantes en situación de discapacidad, además de promover } \\
\text { en los docentes la aplicación del diseño universal de aprendiza- } \\
\text { je (entre otros objetivos) (PUCV, 2017) }\end{array}$ \\
\hline $\begin{array}{l}\text { Universidad de Santiago de } \\
\text { Chile (Santiago) }\end{array}$ & $\begin{array}{l}\text { Programa de Acceso Inclusivo, } \\
\text { Equidad y Permanencia }\end{array}$ & $\begin{array}{l}\text { - Ofrecer oportunidades de nivelación, acompañamiento aca- } \\
\text { démico y aprendizaje a los estudiantes que ingresan mediante } \\
\text { vías de acceso inclusivo e ingreso regular, por medio de tres } \\
\text { modalidades de trabajo: tutorías, asesorías y talleres. Además, } \\
\text { se ofrece apoyo y orientación en otros aspectos que pueden } \\
\text { influir en el rendimiento académico (USACH, 2017) }\end{array}$ \\
\hline $\begin{array}{l}\text { Universidad Austral de Chi- } \\
\text { le (Valdivia, Puerto Montt y } \\
\text { Coyhaique) }\end{array}$ & UACh Inclusiva & $\begin{array}{l}\text { - Generar un sistema de apoyo académico a estudiantes con dis- } \\
\text { capacidad, para favorecer su inclusión educativa, potenciando } \\
\text { y nivelando sus oportunidades de aprendizaje y contribuyendo } \\
\text { a su graduación oportuna. También, establecer y desarrollar } \\
\text { actividades de vinculación con la comunidad universitaria y } \\
\text { con el medio local en iniciativas que promuevan la inclusión } \\
\text { educativa, entre otros objetivos (UACh, 2017) }\end{array}$ \\
\hline $\begin{array}{l}\text { Universidad Católica del } \\
\text { Norte (Antofagasta y } \\
\text { Coquimbo) }\end{array}$ & Programa Incluye UCN & No informa \\
\hline
\end{tabular}

Rodríguez / Valenzuela. Acceso y permanencia de estudiantes con discapacidad en las universidades chilenas Sinéctica 53 www.sinectica.iteso.mx 


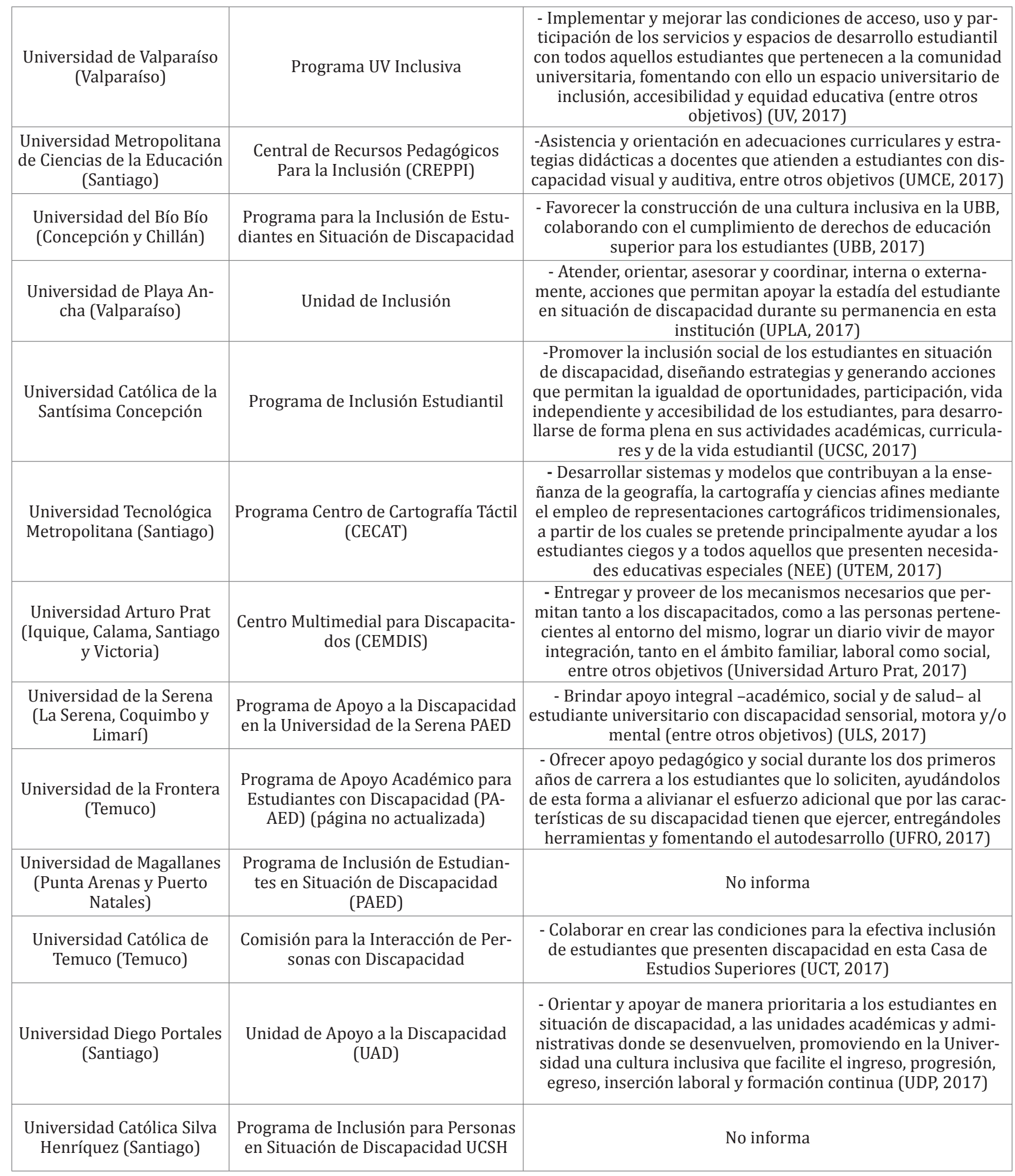

Fuente: Elaboración propia a partir de páginas electrónicas institucionales. 